\title{
Formación e innovación en las empresas industriales ${ }^{1}$
}

\section{Isabel García Espejo}

Universidad de Oviedo. Departamento de Economía Aplicada igarcia@uniovi.es

\section{Resumen}

El objetivo de este artículo es analizar las relaciones entre la formación y la innovación dentro de las empresas industriales. El trabajo se inicia con la exposición de los factores que, en las investigaciones realizadas al efecto, se han encontrado más asociados con ambas estrategias empresariales. El análisis empírico se basa en la información obtenida por medio de una encuesta realizada a las industrias de más de treinta trabajadores ubicadas en el Principado de Asturias. Mediante técnicas de regresión logística, se analizan las relaciones entre determinados aspectos de la formación (tasa de cobertura, detección de necesidades, tipo de formación, organización de las acciones formativas, evaluación y valoración de la formación) y la actividad innovadora. Los resultados muestran una fuerte asociación entre ambas prácticas empresariales, así como diferencias significativas en la implementación y valoración de la formación según el grado de innovación de las empresas.

Palabras clave: formación en la empresa, innovación, estrategias empresariales, sector industrial.

\section{Abstract. Training and innovation in industrial enterprises}

The aim of this article is to analyze the relationship between training and innovation in industrial enterprises. It starts with an account of the factors that corresponding research has shown to be most strongly related to both business strategies. The empirical analysis is based on the outcome of a survey carried out in industries located in the Principality of Asturias with more than 30 employees. By means of logistic regression techniques we analyze the relations between certain aspects of training (level of coverage, detection of necessities, type of training, organization of training activities, evaluation and valuation of training) and innovative activities. The results show a strong association between both business practices, as well as significant differences in the implementation and valuation of training depending on the level of innovation in firms.

Key words: workplace training, innovation, business strategies, industrial sector.

1. Este artículo se integra dentro de una investigación financiada por el Plan Nacional de I+D+I del Ministerio de Educación y Ciencia denominada Sociedad del conocimiento y nuevos modelos de organización social del trabajo (Proyecto SEC2002-03009). 


\section{Sumario}

Introducción Las relaciones entre formación

Factores asociados con la formación y la innovación en las empresas e innovación

Formación e innovación en la industria asturiana

Conclusiones

Bibliografía

\section{Introducción}

La formación y la innovación constituyen estrategias empresariales que están cobrando especial importancia en el entorno actual en el que tienen que desenvolverse las organizaciones. El endurecimiento de la competitividad, la globalización de la economía, el rápido cambio tecnológico y la exigencia de una mayor calidad en la producción de bienes y servicios constituyen elementos que obligan a las empresas a adoptar estrategias innovadoras si quieren competir con éxito en el mercado. Una situación que difiere de épocas anteriores, no muy lejanas, cuando las empresas basaban su supervivencia en el aprovechamiento de las economías de escala y en el logro de altos niveles de productividad a costes reducidos (Huerta Arribas, 2003).

En este entorno altamente competitivo, la formación viene a ser también un elemento clave, especialmente en las empresas innovadoras que exigen de sus recursos humanos un constante aprendizaje de nuevas competencias. Sin embargo, pese a su creciente importancia, la formación todavía no está suficientemente implantada en las empresas españolas, muchas de las cuales consideran que los recursos económicos destinados a acciones formativas constituyen más un gasto que una inversión. Sirva como indicador de esta afirmación la posición claramente inferior de España en inversión formativa respecto a países como Alemania, Dinamarca, Noruega y Holanda (Sáez, 2002). Así, estudios recientes sitúan el esfuerzo en formación que realiza la empresa española en una media de 21,15 horas por trabajador y año, frente, por ejemplo, a las cincuenta horas de Alemania (Huerta Arribas, 2003).

El panorama que ofrece la innovación en nuestro país tampoco es muy alentador. En España, el gasto en I+D se sitúa por debajo de la media europea y la innovación se concentra todavía en un número reducido de comunidades autónomas (Calvo, 2000). Además, los estudios realizados al efecto indican una elevada dependencia exterior por parte de la industria española, como así lo atestiguan el bajo nivel de gastos en comercialización de nuevos productos y el alto porcentaje de gasto en patentes y licencias de uso (Pradas Poveda, 1995). A la inversa, la protección que logran las empresas para sus innovaciones por medio de patentes es muy reducida (Guarnizo García y Guadamillas Gómez, 1998).

No obstante, pese a estos datos poco halagüeños, cada vez son más las empresas que consideran la innovación como una de las estrategias empresariales más efectivas para competir con éxito en un mercado en el que se ofrecen pro- 
ductos y/o servicios similares. De igual forma, cada vez es mayor el interés de las organizaciones por los temas formativos. Se considera que tanto la formación de los recursos humanos como la innovación desempeñan un papel fundamental en la competitividad de las empresas.

Se trata, además, de dos estrategias empresariales que deben estar muy relacionadas entre sí, ya que, al elevarse los niveles de cualificación de los trabajadores, se potencia asimismo la actividad innovadora. De igual forma, si una empresa quiere innovar, tanto en sus procesos productivos como en sus productos o servicios, la formación tiene que constituir una parte del plan estratégico organizacional, dada la necesaria puesta al día de los conocimientos y las capacidades de sus recursos humanos. Sin embargo, aunque es previsible la existencia de una relación entre la formación y la innovación, este vínculo no ha sido suficientemente analizado desde el punto de vista teórico y empírico. En este artículo se aborda precisamente la relación existente entre las estrategias formativa e innovadora de las empresas. Aquí conviene precisar que no se trata de analizar los efectos de la introducción de nuevas tecnologías en los niveles y las necesidades de cualificación de los trabajadores, un tema examinado en múltiples estudios, sino de analizar las diferencias en la provisión, gestión y concepción de la formación según el grado de innovación de las empresas. La fuente fundamental de datos es una encuesta realizada a las industrias asturianas de más de treinta trabajadores.

La estructura de este artículo es la siguiente. En el primer apartado, se exponen los principales factores que, en las investigaciones realizadas al efecto, se han encontrado más asociados con la formación y la innovación en las empresas, tanto desde el punto de vista teórico como empírico. El segundo apartado está dedicado al estudio de la formación y la innovación en la industria asturiana, así como al análisis de las relaciones entre la formación y la innovación dentro de las empresas. Finalmente, en el apartado tercero, se exponen las conclusiones más importantes del artículo.

\section{Factores asociados con la formación y la innovación en las empresas}

Son numerosos los estudios que han analizado los factores que se hallan asociados al esfuerzo formativo realizado por las empresas, así como los efectos que tiene la formación sobre los trabajadores y sobre determinados resultados organizacionales. A este respecto, existe una abundante literatura que relaciona la formación con incrementos significativos de la productividad laboral (Black y Lynch, 1996; Barret y O'Connell, 2001; Kraiger, McLinden y Casper, 2004). También se ha encontrado relación con otros resultados organizativos tales como la mejora de la calidad (Kidder y Rouiller, 1997), el incremento de los resultados financieros (D’Arcimoles, 1997) y la reducción de los costes (Aragón Sánchez, 2004).

Ciertas características de las empresas han resultado ser, asimismo, variables significativas en la explicación de la provisión de formación. El tamaño constituye uno de los factores más nombrados. Se considera que las grandes 
empresas realizan un mayor número de acciones formativas al tener estructuras más formalizadas, contar con mercados internos de trabajo y sufrir una menor rotación laboral (Knoke y Kalleberg, 1994; Lynch y Black, 1998). Estrechamente relacionado con el tamaño, figura la presencia de sindicatos como un elemento que favorece la formación de los trabajadores (Frazis, Herz y Horrigan, 1995; Lynch y Black, 1998). Asimismo, el sector productivo en el que opera la empresa constituye un elemento explicativo de las diferencias en la incidencia y gestión de la formación (Frazis, Herz y Horrigan, 1995; Alba Ramírez y Tugores Ques, 2000; García Espejo, 2004). A estos factores, habría que añadir otros pertenecientes al contexto externo de la empresa, tales como la creciente complejidad del entorno, la competitividad del mercado y el marco institucional que regula las relaciones de empleo en cuanto a derechos de antigüedad, sistemas de remuneración, legislación contractual y derechos de pensión (Knoke y Kalleberg, 1994; Greenhalgh y Mavrotas, 1994).

Además de los factores que se asocian con una mayor formación y de su incidencia en los resultados de las empresas, otras aportaciones han abordado los efectos que produce sobre los trabajadores. Especial mención merece la asociación entre la formación y la estabilidad laboral. Se considera que aquellas organizaciones que intentan promover relaciones a largo plazo con sus empleados y reducir la rotación son más proclives a establecer programas formativos (Frazis, Herz y Horrigan, 1995; Knoke y Kalleberg, 1994; García Espejo, 1999). Junto con la estabilidad, también se han señalado otros efectos positivos en el personal de la empresa, tales como el logro de una mayor motivación y satisfacción en el trabajo, así como un aumento de las posibilidades de promoción (Kider y Rouiller, 1997; Kirkpatrick, 1997; García Espejo, 1999).

Si nos referimos ahora a la innovación, lo primero que se debe clarificar es qué se entiende por innovación. Una definición bastante aceptada es aquélla que presenta a la innovación como el resultado de un conjunto de actividades que transforman una idea o una invención en un producto, un servicio o un proceso que sea comercializable y que origine una mejora cualitativa de la oferta existente (Costa, Duch y Lladós, 2001, p. 54). Ahora bien, la innovación no debe confundirse con la ciencia y la tecnología y no debe quedar limitada a la innovación tecnológica orientada a nuevos productos y procesos; a esta definición habría que añadir innovaciones organizativas e innovaciones comerciales (Esteban, Coll y Blasco, 2005).

Tampoco se debe confundir la innovación con la I+D. Desde hace algunos años, el propio Instituto Nacional de Estadística, siguiendo las directrices del Manual de Oslo, utiliza un concepto mucho más amplio de la innovación que el constreñido a la I+D. Así, junto a los gastos en $\mathrm{I}+\mathrm{D}$, se incluyen gastos en la adquisición de maquinaria y en tecnología inmaterial y de software; gastos en diseño y en ingeniería industrial; desarrollo de instalaciones para producir experimentalmente y luego normalmente; los gastos de formación y de comercialización; la reorganización de los sistemas de gestión y de producción; así como los métodos, por ejemplo: nuevos métodos de gestión de estocs y de control de calidad (INE, 2004). 
Hecha esta precisión, el análisis de los determinantes de la actividad innovadora de las empresas se ha centrado, en su mayor parte, en factores de carácter externo, como el sector de actividad, así como en el tamaño de la empresa (Galende del Canto y Suárez González, 1998). Ya en los primeros estudios que plantean este tema, se considera que las grandes empresas son más innovadoras en términos absolutos que las pequeñas, por diversos motivos (Schumpeter, 1950). En primer lugar, porque están mejor capacitadas para aprovechar economías de escala en $\mathrm{I}+\mathrm{D}$, tienen un mayor acceso a la financiación externa y pueden desarrollar varios proyectos a la vez diversificando los riesgos. No obstante, sobre este punto, las investigaciones más recientes no ofrecen resultados concluyentes (Gumbau Albert, 1997). Así, si bien parece que existe una relación entre el tamaño de la empresa y el desarrollo de actividades innovadoras, si hablamos en términos relativos, el esfuerzo innovador es superior en las empresas de menor tamaño, sobre todo las medianas (Guarnizo García y Guadamilllas Gómez, 1998; Fariñas y Huergo, 1999).

Si se consideran los efectos de la innovación en determinados resultados de las empresas, algunas investigaciones han señalado su relación con los incrementos de la productividad, la competitividad e incluso el bienestar social (Busom, 1993; Galán Crespo y Velázquez Angona, 1999). Sin embargo, son menos los estudios que han abordado su relación con ciertas políticas de recursos humanos tales como la formación, y, cuando así ha sido, la actividad formativa se ha analizado junto con otros factores y no de forma prioritaria. Estas aportaciones señalan que una insuficiente formación constituye una restricción a la hora de desarrollar una estrategia innovadora (Huerta Arribas, 2003). Asimismo, se observa una asociación positiva entre las empresas más innovadoras y la provisión de una mayor formación a los trabajadores (Greenhalgh y Mavrotas, 1996; Frazis, Gittelman y Joyce, 2000; Huergo, 2002).

Pese a las referencias citadas, se puede afirmar que todavía no existen suficientes estudios empíricos que hayan abordado el problema con profundidad. Es por ello que pueden ser relevantes aquellos esfuerzos dirigidos a clarificar más las relaciones entre estas dos estrategias empresariales. Con este objetivo, en este artículo se han abordado las relaciones entre formación e innovación en las empresas industriales. Se ha elegido el sector industrial dado que es el sector más estable en sus inversiones, el que tiene mayor capacidad de absorción del progreso tecnológico y, sobre todo, porque en él radica la mayor parte de la producción de innovaciones (Molero y Buesa, 1995; Barba Aragón, Aragón Sánchez y Sanz Valle, 2000). Como espacio territorial del estudio, se ha considerado un ámbito regional, en concreto, el Principado de Asturias, pues se cree conveniente analizar las actividades innovadoras tomando las comunidades autónomas como unidades de análisis ante la gran disparidad regional que existe en España en cuanto a innovación, y la profunda interrelación de este tipo de actividades con el entorno en el que se realizan (Buesa y otros, 2002b; Costa, Duch y Lladós, 2001). 


\section{Formación e innovación en la industria asturiana}

En este apartado, se va a analizar la situación de la formación y de la innovación en el sector industrial asturiano, así como las relaciones entre ambas dimensiones. Como fuente de datos, se ha acudido a la información obtenida por medio de una encuesta realizada en 2005 a empresas industriales asturianas de más de treinta trabajadores. Según el Anuario-Guía de empresas de Asturias (2004), se contabilizaron un total de 280 industrias de más de 30 trabajadores $^{2}$ y se obtuvieron datos de 200 , lo que representa un porcentaje muy elevado de respuesta ${ }^{3}$, el 71,4\%. Las encuestas fueron realizadas a los directores de las empresas o, si procedía, a los responsables de recursos humanos.

En el caso de la formación, esta encuesta nos permite tener una visión mucho más amplia que la que nos facilitan los datos procedentes de la oferta de formación subvencionada (Fundación Tripartita para la Formación en el Empleo) u otras fuentes estadísticas. Igualmente ocurre en el caso de la innovación con la información del Instituto Nacional de Estadística en su desagregación por comunidades autónomas. Además, la utilización de esta encuesta posibilita analizar la relación entre ambas dimensiones, la formación y la innovación dentro de las empresas.

En primer lugar, hay que señalar que el $91 \%$ de las industrias encuestadas ha impartido formación en los últimos años, bien sea directamente o a través de organismos externos, y un $86,5 \%$ ha organizado algún tipo de cursos para sus trabajadores en el último año. Se trata de unas cifras nada despreciables que indican la creciente importancia de la formación, sobre todo en aquellas industrias cuyas plantillas superan los treinta trabajadores.

Si nos referimos al estado de la innovación en Asturias, hay que decir que, a pesar de que se perciben signos de cambio, la propensión empresarial a la innovación es baja (Vázquez y Lomba, 2000). Si atendemos a los gastos en $\mathrm{I}+\mathrm{D}$ y en innovación del sector industrial según comunidades autónomas, que aparecen en la tabla 1, se observa claramente como Asturias no figura a la cabeza de tales gastos. Su participación en I+D y en innovación suponen, respectivamente, un $1,3 \%$ y un $1,1 \%$ de los gastos totales nacionales.

Asturias tampoco cuenta con un sistema regional de innovación suficientemente articulado. Se puede definir un sistema regional de innovación como «un conjunto de redes entre agentes públicos y privados que interactúan y se retroalimentan en un territorio específico, aprovechando una infraestructura propia para los propósitos de adaptar, generar y difundir conocimientos e innovaciones» (Buesa y otros, 2002a, p. 16). Los agentes de este sistema son las empresas, la infraestructura de soporte a la innovación, los organismos y

2. Se consideró conveniente establecer un umbral mínimo de trabajadores y eliminar así al gran número de empresas de muy pequeño tamaño, en las que es difícil que se den estrategias formativas o de innovación de cierta entidad.

3. En un principio, según el Anuario-Guía (versión 2004) se contabilizaron 351 empresas, de las que 71 fueron rechazadas con posterioridad a razón de su activiad no industrial (62) o por tratarse de organizaciones duplicadas (9). 
Tabla 1. Gastos en I+D y gastos en innovación por comunidades autónomas (2004) (en miles de euros)

\begin{tabular}{lrrrc}
\hline & \multicolumn{1}{c}{$\begin{array}{c}\text { Gastos } \\
\text { en I+D }\end{array}$} & $\begin{array}{l}\text { Gastos } \\
\text { en I+D (\%) }\end{array}$ & $\begin{array}{l}\text { Gastos } \\
\text { en innovación }\end{array}$ & $\begin{array}{l}\text { Gastos } \\
\text { en innovación (\%) }\end{array}$ \\
\hline Total & 8.945 .761 & 100,0 & 12.490 .813 & 100,0 \\
Andalucía & 882.913 & 9,9 & 1.182 .776 & 9,5 \\
Aragón & 180.045 & 2,0 & 463.409 & 3,7 \\
Principado de Asturias & 116.252 & 1,3 & 142.259 & 1,1 \\
Illes Balears & 54.687 & 0,6 & 80.729 & 0,6 \\
Canarias & 199.285 & 2,2 & 139.085 & 1,1 \\
Cantabria & 46.158 & 0,5 & 57.177 & 0,4 \\
Castilla y León & 423.081 & 4,7 & 487.700 & 3,9 \\
Castilla-La Mancha & 116.589 & 1,3 & 235.108 & 1,9 \\
Cataluña & 2.106 .870 & 23,6 & 3.073 .661 & 24,6 \\
Comunidad Valenciana & 731.940 & 8,2 & 804.510 & 6,4 \\
Extremadura & 56.947 & 0,6 & 59.383 & 0,5 \\
Galicia & 366.290 & 4,1 & 534.366 & 4,3 \\
Comunidad de Madrid & 2.447 .465 & 27,3 & 3.552 .955 & 28,4 \\
Región de Murcia & 138.267 & 1,5 & 170.476 & 1,4 \\
Navarra & 256.947 & 2,9 & 255.368 & 2,0 \\
País Vasco & 778.443 & 8,7 & 1.186 .154 & 9,5 \\
La Rioja & 41.173 & 0,5 & 57.652 & 0,5 \\
\hline
\end{tabular}

Fuente: estadística sobre actividades de I+D (2004), INE. Encuesta sobre innovación tecnológica en las empresas (2004), INE. Elaboración propia.

las actuaciones públicas vinculadas a la innovación y el entorno regional y productivo de la innovación. Pues bien, se echa en falta más coordinación entre los agentes y la interacción entre sus actividades, así como una mayor conexión entre la investigación y las necesidades y los objetivos de las empresas (Fernández y Vázquez, 1996; Vázquez y Lomba, 2000).

No obstante, sin negar el importante papel que desempeña la Administración, de todos los elementos del sistema regional de innovación, las empresas son el subsistema de mayor importancia, sobre todo las que son innovadoras, al facilitar la conexión entre el sistema productivo y el de innovación (Buesa y otros, 2002a). Asturias presenta ciertas particularidades en cuanto al tipo de empresas más innovadoras. En la región asturiana, se estima que el $75 \%$ de las empresas involucradas en actividades de I+D interna tienen menos de 250 trabajadores, en contraste con el total nacional, donde las PYME sólo realizan el 46\% del gasto (PCTI Asturias 2006-2009).

$\mathrm{Si}$ atendemos a los datos de la encuesta a empresas industriales, cerca de un $53 \%$ de las industrias han desarrollado alguna innovación. En torno a un 
$29 \%$ las han patentado, lo que significa un número relativamente bajo de empresas que viene a confirmar que la protección de la actividad innovadora por medio del registro de patentes se realiza en pocas ocasiones en España (Guarnido García y Guadamillas Gómez, 1998). Por último, la colaboración externa para proyectos conjuntos de innovación con otras organizaciones tampoco es una práctica que esté muy extendida entre las industrias. Del total de empresas, sólo el $27,4 \%$ ha colaborado con otras entidades para actividades de $\mathrm{I}+\mathrm{D}+\mathrm{I}$.

\section{Las relaciones entre formación e innovación}

El análisis empírico realizado en este apartado para determinar las relaciones entre la formación y la innovación dentro de las organizaciones se basa en la encuesta a empresas industriales realizada en 2005. Para analizar dicha relación, es necesario seleccionar un indicador que nos mida el grado de innovación en dichas industrias.

Se han utilizado diversos indicadores para medir la innovación en la literatura dedicada a este tema. La mayor parte de los estudios se han centrado en las empresas que desarrollan actividades de I+D. Ahora bien, la utilización de los gastos en $\mathrm{I}+\mathrm{D}$ como indicador de la innovación presenta varias y serias limitaciones. Por un lado, se subestiman la actividad innovadora de las pequeñas empresas, ya que numerosas empresas innovan con muy pocos recursos para $\mathrm{I}+\mathrm{D}$, a la vez que se obtienen resultados claramente sesgados a favor de la gran empresa. Por otro, se elimina cualquier referencia a otras actividades innovadoras que cada vez tienen mayor importancia. Así, las empresas pueden desarrollar otras estrategias de innovación, como son la adquisición de bienes de equipo, la realización de actividades dirigidas a la mejora de productos y procesos productivos o el desarrollo de tecnologías vinculadas al procesamiento de la información que no requieren de procesos de $\mathrm{I}+\mathrm{D}$, sino la subcontratación de empresas de servicios (Urraca Ruiz, 1998; INE, 2004).

Además, los gastos en $\mathrm{I}+\mathrm{D}$ sólo representan una parte de los gastos que lleva consigo el proceso de innovación. Hasta hace poco tiempo se consideraba que la innovación constituye un proceso en cadena del que las actividades de I+D son el primer escalón. Esta idea ha sido ya abandonada, y en la actualidad se utiliza un concepto mucho más amplio de la innovación, como es el utilizado por parte del INE, siguiendo las líneas marcadas por el Manual de Oslo (Calvo, 2000; INE, 2004).

Una forma alternativa de medir la innovación a los gastos de $\mathrm{I}+\mathrm{D}$ es la utilización de las patentes. Sobre este indicador, existe una gran controversia y ha sido objeto de frecuentes críticas. Se considera que las empresas pueden elegir otras formas de protección de sus invenciones que el sistema de patentes. Asimismo, hay que tener en cuenta las diferentes propensiones a patentar algunas características determinadas, como el tamaño de la empresa, los sectores industriales, las regiones e incluso los países (Coronado Guerrero y Acosta Seró, 1999; Buesa y otros, 2002b). 
Junto a estos indicadores, hay que señalar otro tipo de medidas utilizadas fundamentalmente por aquellos estudios que han generado sus propios datos a partir de encuestas a empresas. En estos casos, es frecuente considerar como indicador si la empresa ha introducido innovaciones, ya sea de producto, de proceso e incluso de organización (Costa, Duch y Lladós, 2001; Huergo, 2002). En esta misma línea, se enmarca el presente artículo. Ahora bien, aunque en la encuesta a empresas industriales en la que se basa este estudio también se pregunta si la empresa ha realizado alguna innovación, se ha estimado más adecuado utilizar otro indicador obtenido a partir de la pregunta sobre la capacidad innovadora de la empresa; en concreto, si se considera que la empresa ha sido hasta el momento más innovadora que la media del sector, con una escala ordinal de 1 a 5 , donde 1 significa "muy en desacuerdo" y 5, "muy de acuerdo». Se estima que esta medida es más acertada que la anterior, centrada en el desarrollo de una innovación que puede ser de carácter puntual, dado que indica una actividad innovadora continuada y consolidada, a la vez que permite una graduación de la respuesta.

Para analizar la relación entre formación e innovación en las empresas industriales, se ha recurrido a un modelo de regresión logística de tipo ordinal, donde la variable dependiente es el indicador de innovación antes mencionado: « $\mathrm{Si}$ se considera que la empresa ha sido hasta el momento más innovadora que la media del sector». Conviene señalar que, en este artículo, no se pretende realizar la cuantificación de probabilidades. La intención de la construcción del modelo se ha centrado en el análisis de la «correlación» o «asociación» de las variables que se han considerado relevantes (tabla 2).

Las dimensiones y variables independientes que han sido incorporadas referentes a la formación han sido ${ }^{4}$ :

- Cobertura de la formación, que se ha medido a partir de tres variables. Una variable dicotómica que mide si la empresa ha organizado formación en los últimos años, bien sea directamente o a través de organismos externos. Otra variable igualmente dicotómica sobre si ha organizado formación on-line, y una tercera variable que mide el porcentaje de trabajadores que han sido formados en el último año (hasta el 25\%, del 26 al 50\% y más del 50\%).

- Categorías laborales formadas. La incidencia de las acciones formativas según los tipos de trabajadores se ha medido a partir de seis variables dicotómicas: personal directivo; mandos intermedios; técnicos universitarios; empleados administrativos y comerciales; trabajadores manuales cualificados, y trabajadores manuales no cualificados.

- Origen de las necesidades de formación. Esta dimensión está representada por ocho variables dicotómicas referidas a diferentes fuentes que pueden intervenir en la detección de las necesidades formativas, y que son: a peti-

4. Se han descartado problemas de multicolinealidad entre las variables. 
ción de los propios empleados; a petición del personal directivo; la oferta de formación subvencionada; los resultados de la evaluación del rendimiento de los trabajadores; análisis de los planes futuros de la empresa; adaptación de la empresa a las nuevas tecnologías; peticiones del Departamento de Recursos Humanos/Formación, o bien a propuesta de los representantes sindicales de la empresa.

- Organización de la formación. Este aspecto de la actividad formativa se ha analizado a través de cuatro variables: porcentaje del personal que imparte la formación que es de la empresa (ninguno, hasta el 50\%, más del $50 \%$, todos); porcentaje de las acciones de formación que se desarrollan en el puesto de trabajo (todas fuera del puesto, hasta el $50 \%$ en el puesto, más del 50\% en el puesto, todas en el puesto); porcentaje de acciones de formación realizadas dentro de la jornada laboral (todas fuera de la jornada, hasta el 50\% durante la jornada, más del 50\% durante la jornada, todas dentro de la jornada). Por último, una variable cuantitativa que mide la duración de los cursos.

- Tipo de formación que se ha impartido. Sobre este punto, en primer lugar, se ha incorporado al análisis una variable ordinal que mide el carácter general o específico de la formación por medio de una escala de 1 a 5 , donde 1 es "conocimientos y habilidades de carácter general» y 5 «conocimientos y habilidades de carácter específico». Se han incluido, asimismo, los tipos de cursos por medio de ocho variables dicotómicas que hacen referencia a la naturaleza de los mismos: vestibular; programas informáticos; formación de formadores; prevención de riesgos laborales; nuevas líneas de producción; mantenimiento; nuevas tecnologías y maquinaria; idiomas.

- Evaluación de la formación. Se incluyen cinco prácticas evaluadoras, cada una de las cuales es una variable independiente dicotómica: el grado de satisfacción de los empleados; los conocimientos y las habilidades adquiridas; la aplicación de conocimientos y habilidades al puesto de trabajo; la mejora de los resultados empresariales; la asistencia y el seguimiento de cursos.

- Por último, se ha considerado la valoración de la formación a partir de cuatro variables con escala ordinal de 1 a 5 , donde 1 significa "muy en desacuerdo" y 5, "muy de acuerdo»: la empresa considera los gastos en formación como una inversión; la empresa forma a sus empleados sólo cuando es imprescindible; la formación se orienta a cubrir necesidades a corto plazo; los empleados participan en la planificación de la formación.

- Además de las variables que tienen que ver con la actividad formativa, se han introducido en el modelo dos variables de control: el tamaño de la empresa (de 30 a 50 trabajadores, de 51 a 100, más de 100) y la antigüedad medida a partir del año de fundación de la organización (hasta 1970, de 1971 a 1980 , de 1981 a 1990 , de 1991 a 2003). En el modelo que figura a continuación, sólo se presentan las variables que han resultado significativas. 
Tabla 2. Modelo de regresión logística ordinal. Relaciones entre formación e innovación

\section{Provisión de formación}

Ha realizado formación en los últimos años (Cat. residual: no)

$18,520^{* *}$

\section{Incidencia de la formación}

Porcentaje de trabajadores formados (Cat. residual: hasta el 25\%)

1. Del $26 \mathrm{Al} 50 \%$

2. Más del $50 \%$

$3,026^{* *}$

\section{Categorías profesionales formadas}

Personal directivo (Cat. residual: no)

Mandos intermedios (Cat. residual: no)

Empleados administrativos y comerciales (Cat. residual: no)

Trabajadores manuales no cualificados (Cat. residual: no)

$10,137^{* * *}$

$-7,544^{* * *}$

$-5,917^{* *}$

$-5,468^{* * *}$

\section{Origen de las necesidades de formación}

A petición del personal directivo (Cat. residual: no)

$3,251^{*}$

Oferta de formación subvencionada (Cat. residual: no)

$-2,942^{*}$

Resultados de la evaluación del rendimiento (Cat. residual: no)

$-4,192^{* *}$

Análisis de los planes futuros de la empresa (Cat. residual: no)

$3,315^{* *}$

Adaptación de la empresa a las nuevas tecnologías (Cat. residual: no)

$6,461^{* * *}$

\section{Organización de la formación}

Porcentaje de las acciones de formación dentro de la jornada laboral

(Cat. residual: todas dentro de la jornada)

1. Todas fuera de la jornada

2. Hasta el $50 \%$ durante la jornada

3. Más del $50 \%$ durante la jornada

Tipo de formación

Nuevas líneas de producción (Cat. residual: no)

\section{Evaluación de la formación}

Grado de satisfacción de los empleados (Cat. residual: no)

$-3,705^{* *}$

Aplicación de los conocimientos y habilidades (Cat. residual: no)

Mejora de los resultados empresariales (Cat. Residual: no)

Asistencia y seguimiento de cursos (Cat. residual: no)

Valoración de la formación

Se consideran los gastos en formación como una inversión (Escala 1 a 5)

$1,197^{* *}$

\section{Antigüedad de la empresa}

Año de fundación de la empresa (Cat. residual: hasta 1970)

1. De 1991 a 2003

2. De 1981 a 1990

3. De 1971 a 1980

2,779

\section{Tamaño de la empresa}

Número de trabajadores de la empresa (Cat. residual: más de 100 trabajadores)

1. De 30 a 50 trabajadores

2. De 51 a 100 trabajadores

\section{Número total de casos $\quad 200$}

Niveles de significación: ${ }^{*} p<0,1000,{ }^{* *} p<0,0500,{ }^{* * *} p<0,0100$.

Fuente: Encuesta a empresas industriales 2005. 
Según los resultados obtenidos, las empresas industriales innovadoras son las que más proveen formación a sus empleados. La realización de formación en los últimos años constituye una variable cuyo coeficiente presenta un peso muy elevado y significativo. Asimismo, son las industrias que presentan una tasa de cobertura de la formación mayor a tenor del porcentaje de trabajadores formados. Respecto a las categorías ocupacionales, es la formación del personal directivo la variable que más se asocia con la innovación. En este punto, los resultados coinciden con otros estudios en los que se observa una fuerte relación entre los sectores innovadores, y que incorporan cambios tecnológicos con mayor frecuencia, y las ocupaciones que implican tareas de organización y gestión de recursos en la empresa (Peraita, 2000).

Si atendemos al origen de las necesidades de formación, figuran asociadas de manera positiva y significativa con una mayor capacidad innovadora la adaptación de la empresa a las nuevas tecnologías, el análisis de los planes futuros de la empresa y las peticiones del personal directivo. Por el contrario, los resultados de la evaluación del rendimiento y la oferta de formación subvencionada no constituyen fuentes a partir de las cuales se generen acciones formativas. Estos resultados indican la mayor relevancia que tienen para las industrias innovadoras las estrategias empresariales (planes futuros) en el diagnóstico de las necesidades de formación de sus recursos humanos.

Respecto al tipo de formación impartida, la variable que mide el grado en que los conocimientos transmitidos son bien de carácter general o bien de carácter específico, no ha resultado significativa. No hay, por tanto, diferencias entre las empresas en este aspecto según su grado de innovación. Sí, en cambio, se observan discrepancias en los cursos concretos. Así, los cursos más frecuentes son precisamente los que tienen que ver con el establecimiento de nuevas líneas de producción, es decir, aquéllos más relacionados con actuaciones innovadoras en los procesos productivos y en los productos.

Si nos referimos ahora a la organización de la formación, ni el tipo de personal que imparte los cursos, ni el lugar donde se van a desarrollar las acciones formativas, ni la duración de los cursos constituyen aspectos en los que se perciba un comportamiento distinto entre las empresas según su grado de innovación, y como tal no resultan variables que logren significación estadística. Sí, en cambio, parece haber diferencias en la implementación de los cursos dentro o fuera de la jornada laboral. Los resultados indican una tendencia en las industrias más innovadoras a que estas acciones se realicen fuera de la jornada laboral, o al menos una parte importante de ellas.

También se aprecian distintos sistemas de evaluación según el grado de innovación de las industrias. Tanto la satisfacción de los empleados, como la asistencia y el seguimiento de los cursos, como la aplicación al puesto de los conocimientos y las habilidades presentan signos negativos en sus coeficientes. El aspecto que más se evalúa conforme la empresa es más innovadora, es precisamente el más difícil de evaluar: la mejora de los resultados empresariales. Este aspecto es, además, el más importante si se considera que la finalidad última de la formación debe ser el aumento de las ventajas competitivas de las 
empresas. Asimismo, también es distinta la valoración de la formación según el grado de actividad innovadora. A este respecto, concebir la formación como una inversión y no como un gasto se asocia de manera positiva con la innovación.

Por último, respecto al resto de variables introducidas en el modelo, son las empresas de 30 a 50 trabajadores y, sobre todo, las de 51 a 100 las que más probabilidades tienen de innovar por encima de la media del sector. Igualmente importante por su significación es la antigüedad de la empresa. Son las industrias más jóvenes, las fundadas después de 1980, las que más probabilidades tienen de introducir innovaciones, tanto en sus procesos productivos como en sus productos.

\section{Conclusiones}

A lo largo de este artículo, se han analizado las relaciones entre la formación y la innovación dentro de las empresas industriales. De los resultados obtenidos, se desprende que ambas prácticas están muy relacionadas entre sí, ya que son las empresas que se caracterizan por su mayor grado de innovación las que ofrecen una cobertura de formación más amplia a sus trabajadores. Se podría incluso afirmar que la formación cumple una función estratégica dentro de estas organizaciones, al potenciar, a través de la cualificación del capital humano, la propia actividad innovadora.

En las empresas innovadoras, son la introducción de nuevas líneas de producción y los planes futuros de la empresa las fuentes principales a partir de las cuales se implementan las acciones formativas, un resultado que apunta nuevamente hacia el carácter estratégico de la formación en este tipo de organizaciones y hacia una gestión más integral de los recursos humanos. Además, tales industrias no dependen de la oferta de formación subvencionada para sus actividades, un hecho que no deja de ser relevante ante el actual sistema de bonificación caracterizado por la cofinanciación de las acciones formativas frente al anterior sistema de subvención. Se trata de un cambio que implica un esfuerzo inversor por parte de las empresas que no todas pueden estar dispuestas a realizar 5 .

La categoría ocupacional sobre la que inciden las acciones formativas en mayor medida, conforme aumenta el grado de innovación, es aquélla más relacionada con la organización y la gestión de los recursos humanos, la categoría de directivos. Ya no se trata, pues, de acciones de reconversión dirigidas preferentemente al personal manual cualificado y a los mandos intermedios a raíz de la introducción de nueva maquinaria, como ocurre en el conjunto de la

5. Real Decreto 1046/2003; Orden TAS/500/2004. En el actual sistema, las empresas disponen de un crédito específico para la formación que es el resultado de aplicar, a sus cotizaciones por formación profesional durante el año anterior, un porcentaje de bonificación que se establece cada año en la Ley de Presupuestos Generales del Estado, en función del tamaño de las empresas. 
industria. En las empresas innovadoras desempeñan un papel central las categorías más altas de la jerarquía ocupacional, aquéllas que, a su vez, pueden potenciar la innovación por su papel decisorio y generador de ideas.

Si se considera que la finalidad prioritaria de la formación es la mejora de los resultados empresariales, este aspecto es el menos evaluado por el conjunto de las industrias. Si embargo, cuanto más innovadora es la empresa, más importancia cobra la fase final de la evaluación; un dato que está relacionado con la valoración de la formación por parte de estas organizaciones. Así, en las industrias innovadoras, la formación es vista como una inversión y no como un gasto, de ahí el énfasis en los resultados empresariales. Es precisamente el análisis del impacto de la formación en aspectos tales como la productividad, la reducción de costes, el aumento de la calidad, etc., lo que confiere el carácter de inversión a la actividades formativas realizadas y posibilita determinar su papel en el logro de las estrategias empresariales.

Por último, cabe señalar que el conjunto de resultados obtenidos permite remarcar la importancia de las empresas innovadoras en la revitalización del tejido empresarial de una región. Todo ello sin olvidar el papel privilegiado que tienen en el propio sistema regional y nacional de innovación. A este respecto, se considera que las empresas innovadoras tienen una mayor influencia en el crecimiento de la producción y en el output tecnológico de una región que el papel desempeñado por la Administración pública y por las universidades (Coronado Guerrero y Acosta Seró, 1999; Buesa y otros, 2002 b). Es por ello que las instancias públicas con competencias en la materia deberían impulsar el surgimiento de empresas que desarrollen actividades de creación de nuevos conocimientos, a la vez que fomentar las redes de cooperación entre todos los elementos del sistema regional de innovación (empresas, universidad, centros tecnológicos y otros agentes); unas relaciones que, como se ha visto en este artículo, no son lo suficientemente intensas en la actualidad.

\section{Bibliografía}

Alba Ramírez, A.; Tugores Ques, M. (2000). «Un estudio microeconómico sobre los determinantes y efectos de la formación en España». En: SÁEZ, F. (coord.). Formación y empleo. Madrid: Fundación Argentaria, p. 341-420.

ARAGÓN SÁNCHEZ, A. (2004). "Gestión de la formación en la empresa». En: MONREAL, J. (dir.). Formación y cultura empresarial en la empresa española. Madrid: Civitas, p. 211-240.

Barba Aragón, M. I.; Aragón Sánchez, A.; Sanz Valle, R. (2000). "Condicionantes de la formación en las PYMES industriales». Economía Industrial, $\mathrm{n}^{\circ} 334$, p. 35-44.

BARRET, A.; O'CONNELL, P. J. (2001). «Does training generally work? The returns to in-company training». Industrial and Labor Relations Review, no 54, 3, p. 647-662.

BLACK, S. E.; LYNCH, L. M. (1996). «Human-Capital Investments and Productivity». The American Economic Review, no 86, 2, p. 263-267. 
Buesa, M.; Martínez, M.; Heijs, J.; BaUmert, T. (2002a). «Los sistemas regionales de innovación en España. Una tipología basada en indicadores económico e institucionales». Economía Industrial, no 347, p. 15-32.

Buesa, M.; Baumert, T.; Heijs, J.; Martínez, M. (2002b). «Los factores determinantes de la innovación: un análisis econométrico sobre las regiones españolas». Economía Industrial, no 347, p. 67-84.

Busom, I. (1993). «Evaluación de los efectos de las subvenciones públicas a las actividades de I+D». Economía Industrial, no 289, p. 141-152.

CALVO, J. L. (2000). «La distribución del gasto empresarial en innovación entre las comunidades autónomas españolas (1994-1998)». Economía Industrial, no 334, p. 71-80.

Coronado Guerrero, D.; Acosta Seró, M. (1999). «Innovación tecnológica y desarrollo regional». Información Comercial Española, no 781, p. 103-116.

Costa, M. T.; DUCH, N.; Lladós, J. (2001). «Determinantes de la innovación y efectos sobre la competitividad: el caso de las empresas textiles». Revista Asturiana de Economía, no 20 , p. 53-80.

D’ARCimoles, C. H. (1997). «Human Resources Policies and Company Performance: A Quantitative Approach Using Longitudinal Data». Organization Studies, no 8, 5, p. 857-874.

esteban García, J.; Coll Serrano, V.; Blasco Blasco, O. M. (2005). "¿Competitividad e innovación en la micro y pequeña empresa? Retos previos a superar». Estudios de Economía Aplicada, no 23, 3, p. 559-581.

FARIÑAS, J. C.; Huergo, E. (1999). «Tamaño empresarial, innovación y políticas de competencia». Economía Industrial, no 329, p. 67-80.

FERNÁNDEZ, E.; VÁZQUEZ, J. A. (1996). «PYMES y política de innovación tecnológica en Asturias». Economía Industrial, no 312, p. 49-55.

FraZIS, H. J.; HerZ, D. E.; HorRigAN, M. W. (1995). «Employer-provided training: results from a new survey». Monthly Labor Review, no 118, 5, p. 3-17.

Frazis, H. J.; GitTleman, M.; JoYCE, M. (2000). «Correlates of training: an analysis using both employer and employee characteristics». Industrial and Labor Relations Review, no 53, 3, p. 443-462.

Galán Crespo, J.; VelázQuez Angona, F. J. (1999). «Principales rasgos de los sectores innovadores en España. Una explotación de la encuesta sobre innovación tecnológica en las empresas». Papeles de Economía Española, no 81, p. 104-111.

Galende Del Canto, J.; SuÁrez GonZÁlez, I. (1998). "Los factores determinantes de las inversiones empresariales en I+D». Economía Industrial, no 319, p. 63-76.

García Espejo, I. (1999). «Formación en el trabajo y movilidad laboral». Papers, no 59, p. 195-219.

- (2004). «Sectores productivos y formación». En: MonREAL, J. (dir.). Formación y cultura empresarial en la empresa española. Madrid: Civitas, p. 105-131.

GreENHALgh, C.; MAVROTAS, G. (1994). «The Role of Career Aspirations and Financial Constraints in Individual Access to Vocational Training». Oxford Economic Papers, $n^{\circ} 46,4$, p. 579-601.

Guarnizo García, J. V.; Guadamillas Gómez, F. (1998). «Innovación y desarrollo tecnológico en las empresas industriales españolas. Factores explicativos según la encuesta de estrategias empresariales». Economia Industrial, no 319, p. 49-62.

Gumbau Albert, M. (1997). "Análisis macroeconómico de los determinantes de la innovación: aplicación a las empresas industriales españolas». Revista Española de Economía, no 14, 1, p. 41-66. 
HuerGO, E. (2002). «Determinantes de la innovación tecnológica en la industria manufacturera española». Revista del Instituto de Estudios Económicos, no 3, p. 121-142.

HuERTA ARribAS, E. (ed.) (2003). Los desafios de la competitividad: La innovación organizativa y tecnológica de la empresa española. Bilbao: Fundación BBVA.

INE (2004). Encuesta sobre innovación tecnológica en las empresas.

KIDDER, P. J.; RouilLER, J. Z. (1997). «Evaluating the Success of a Large-Scale Training Effort». National Productivity Review, primavera, p. 79-89.

KIRKPATRICK, D. L. (1999). Evaluación de acciones formativas: Los cuatro niveles. Barcelona: EPISE.

KNOKe, D.; KalleberG, A. L. (1994). "Job Training in U.S. Organizations». American Sociological Review, no 59, p. 537-546.

KRAiger, K.; MClinden, D.; CASPER, W. J. (2004). "Collaborative planning for training impact». Human Resource Management, no 43, 4, p. 337-351.

LYNCH, L. M.; BLACK, S. E. (1998). «Beyond the incidence of employer-provide training». Industrial and Labor Relations Review, no 52, 1, p. 64-81.

Molero, J.; BuesA, M. (1995). "Configuración productiva y capacidad de innovación en la industria española». Información Comercial Española, no 743, p. 59-83.

PCTI Asturias 2006-2009. <www.pctiasturias.com>.

Peraita, C. (2000). «Características de la formación en la empresa española». Papeles de Economía Española, no 86, p. 295-307.

PRADAS POVEDA, J. I. (1995). «Análisis de la innovación en la empresa industrial española». Economía Industrial, no 301, p. 153-166.

SÁEZ, F. (2002). «Capital humano, innovación y empleo. Su adecuación en el caso español». Economía Industrial, no 348, p. 67-76.

SChumpeter, J. A. (1950). Capitalism, Socialism and Democracy. NY: Harper.

URRACA RUIZ, A. (1998). «I+D y recursos alternativos a la innovación en la industria española». Economía Industrial, no 319, p. 91-104.

VÁZQUEZ, J. A.; LOMBA, R. (2000). «La industria asturiana, un sector en transformación». Economía Industrial, no 335/336, p. 111-122. 